\section{Computer production of punched paper tapes for controlling experiments}

\author{
WALTER vom SAAL*, Princeton University, Princeton, New Jersey 08540 \\ and \\ ROBERT vom SAAL, Gibbs and Cox, Inc., New York, New York 10006
}

Controlling experiments with paper-tape readers often requires the production of complex paper tapes. A program was written to allow automatic production and checking of complex punched paper tapes using a small computer. The user first defines the punches or punch sets to be used. He then specifies, for each successive block of tape to be punched, the number of punch sets of each type to occur and whether those punch sets should be copied in order or automatically randomized. The specified tape is then produced using a two-pass procedure, after which the tape may be automatically checked for punching errors if desired. The program has facilities for retention or modification of user commands from previous runs, simplifying the production of a large number of tapes with similar characteristics.

Many scientific laboratories employ control equipment utilizing punched paper-tape readers. For example, a large number of psychological laboratories use relay or solid-state equipment to present experimental events, with the sequence and timing of these events controlled by a paper-tape reader. As the complexity of experiments and the sophistication of control equipment has increased, a major limitation on the sorts of experiment that can be run has become the feasibility of producing and checking paper tapes of the length and complexity required. In many laboratories, paper tapes are punched one character at a time using a punch kept for that purpose, a method that is laborious, prone to error, and simply impossible to use when tapes of considerable complexity are required.

One solution to the problem of making complex paper tapes is to make them with the aid of a computer. Small computers that can directly punch paper tape are becoming more and more common, and many laboratories could gain occasional access to such a computer. The present article describes a program written to allow the automatic production and checking of complex paper tapes by such a computer.

\section{FACILITIES}

The program may be run on Digital Equipment Corporation PDP-8 or PDP-12 computers. Only the basic computer with Teletype is required, but program options allow the use of a high-speed paper-tape reader and/or high-speed paper-tape punch if available.

* Requests for reprints should be sent to Walter vom Saal, Department of Pschology, Princeton University, Princeton, New Jersey 08540.

\section{PROGRAM OPERATION}

The program is best described by summarizing the several steps involved in using it. After loading the program, the user must (1) define the required punch sets, (2) enter the set-order commands, (3) produce the specified tape in two passes, and (4) check the tape if desired.

\section{Defining Punch Sets}

In this section of the program, the user defines the punch sets he will use. As the computer types out each consecutive set name ( $\mathrm{AA}, \mathrm{AB}$, etc.), the user responds with one or more octal numbers specifying the punches to occur in that set, followed by a carriage return after the last octal number. For example, suppose the user wants to repeatedly use a set of three punches, the first punch having a hole in Column 1 , the second having holes in Columns 1 and 2 , and the third having holes in Columns 1,2 , and 3 . In this case, when the computer types "AA," the user types " $1,3,7$ [carriage return]." In the next section of the program, each time the user wants this particular punch set to be punched, he refers to it by the name "AA."

Each punch in a punch set is defined by typing a single octal number so that punches are not restricted to ASCII characters. A punch set may contain from 1 to more than 100 separate punches, and up to 120 punch sets may be defined. There is a restriction, however, on the total number of punches that may be specified in this section of the program.

This section of the program may be omitted if definitions from previous program runs are to be retained. In addition, a number of special commands allow the user to cancel incorrectly entered definitions, retain, or partially modify certain definitions from previous program runs. When all required punch sets have been defined, the user types an exclamation point to move on to the next section of the program.

Entering Set-Order Commands

In this section the user enters commands indicating the punch sets that should be punched in successive blocks of tape. He also indicates whether or not to randomize the punch sets in each block. For example, to randomize 5 Type AA punch sets and 10 Type $A B$ punch sets, the user types:

\section{AA, 10AB, * (carriage return).}

To punch directly 5 Type AA punch sets followed by 10 Type AB punch sets, the user types:

\section{$5 \mathrm{AA}, 10 \mathrm{AB}$, (carriage return).}

Successive blocks of punch sets may be of different length and may contain different punch sets, and each block may be either randomized or copied directly.

This section of the program may be omitted if previous set-order commands are to be retained. This is useful, for instance, in producing several tapes with the same blocks of randomized punch sets, but with different random orders. After all set-order commands have been entered, the user types an exclamation point to move on to the next section of the program.

\section{Producing Tapes}

At this point, the program produces two tapes. The first contains a complete list of all punch sets in the final randomized order. As this first tape is produced, the set names are typed on the Teletype as a record of the random orders that have been generated. When the first tape is finished, it is read back into the computer and used by the program to produce a second tape containing the actual punch sets in the order specified.

\section{Checking Tapes}

The program has facilities to check both tapes for punching errors. The first tape is automatically checked for the correct total number of punch sets of each type when it is read back in to produce the second tape. The second tape may be fully checked if desired by reading it back in along with the first tape in a final pass of the program.

\section{Example of Program Use}

To illustrate use of the program, the Teletype output of a sample run and 
NEN DEFINITION OF SETS? ( $Y$ OH $N$ ) $Y$

$A A=0,1,3,7 !$
$A B=40 \cdot$
$A C=200 !$
$A D=7,3,1,0 !$

NEW SET ORDER? (Y OR. N) $Y$

3AA,

$10 \mathrm{AB}, 10 \mathrm{AC}, *$

3AD,

$!$

SET-ORDER TAPF ON H OR L PCH? L

$A A, A A, A A$,

$A B, A C, A C, \triangle C, A B, A B, A B, A C, \triangle C, A C$,

$A B, A B, A B, A B, A C, A C, A B, A C, A C, A B$,

$A D, A D, A D$,

!

SET-ORDER TAPE ON H OR L RDR? L

OBJECT TAPE ON H OP L PCH? L

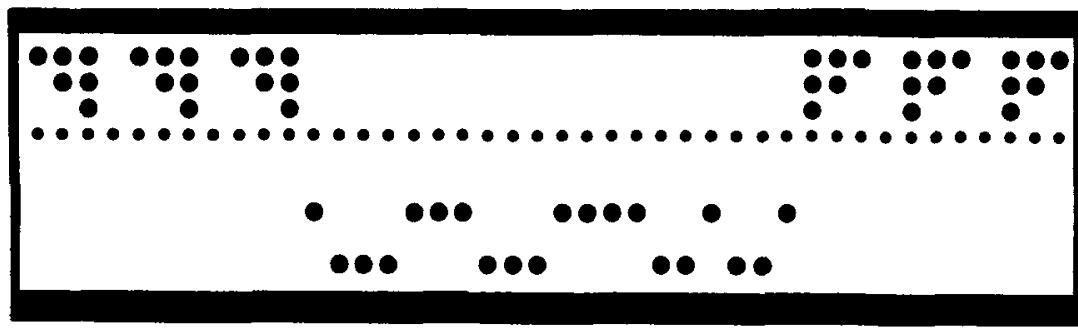

Fig. 1. Teletype output illustrating use of the program. User responses are underlined. The final tape produced is shown beneath the Teletype output.

the final tape produced are shown in Fig. 1. User responses on the Teletype are underlined.

\section{RANDOMIZATION}

There are two general procedures by which a series of events may be randomized. Consider a case where an
E wants to present either Event A or Event B on each trial, randomly, for a total of $\mathbf{1 0 0}$ trials. The first procedure he could use would be to select either Event A (with probability .5) or Event B (with probability .5) on each trial, and repeat this for 100 trials. This would produce a random sequence in the sense that the particular event occurring on each trial could not be predicted from the events that happened on previous trials. However, it would have the possible disadvantage that the number of events of each type would not be fixed but would fluctuate from one sequence to the next. If the $\mathrm{E}$ wanted to have $50 \mathrm{As}$ and $50 \mathrm{Bs}$ in each sequence, he would use the second general randomization procedure, which is simply to select 50 As and $\mathbf{5 0}$ $B s$ and rearrange them into a random order. This procedure is equivalent to random sampling from a pool of $50 \mathrm{As}$ and $50 \mathrm{Bs}$, without replacement. When it is used, the occurrence of a particular event on Trial $n$ is not independent of previous events: on the average, long runs of events of one type will be followed by an increased probability of events of the other type, and a $S$ keeping track of what events occurred on the first 99 trials could predict perfectly what would occur on the 100th trial (if he knew the characteristics of the sequence). However, a sequence produced by using this procedure is still random in the sense that the particular permutation of events used is sampled at random from all the possible permutations. It was this second general randomization procedure that was used in the present program.

One technique sometimes used to randomize the order of $\mathrm{N}$ items in a compute;program is to (1) list the $N$ items in one memory bank, (2) generate a random number associated with each item and list these in a second memory bank, and then (3) copy the original $\mathrm{N}$ items into a third memory bank in a new order determined by ranking the random numbers associated with each item. The disadvantage of this procedure is that $3 \mathrm{~N}$ memory locations are required. The "random swapping" procedure used in the present program was slightly more complicated, but required only $\mathrm{N}$ memory locations. This procedure involved random sampling without replacement from a continually shrinking list. For each item, $i$, between 1 and $N$, a random number, $r$, between $i$ and $N$ was generated and Item $r$ was exchanged with Item $i$. The value of $i$ was then incremented, and the process continued. At each point in this process, the items between $\mathrm{i}$ and $\mathrm{N}$ were the items remaining in the original list, and the random number, $r$, indicated which of those items was sampled. By exchanging Item $r$ with Item $i$ at each point, it was possible to store the new list of items in the space abandoned as the original list was reduced in size, so that a total of only $\mathrm{N}$ memory locations was required. 
Each time a user command indicated that randomization was to occur in the present program, this random swapping procedure was repeated 16 times on the list of punch sets specified. Repetition of the swapping procedure was intended to reduce the effect of whatever biases might exist in the random number generating program. The random number generator was based on one described by Brady (1965) and found to perform satisfactorily according to a number of statistical tests (Brady, 1965; Griffith, 1969). Brady's program was modified to run on the PDP-8 and to transform the numbers generated by his program into numbers between 1 and $k$, where $k$ could be set to any desired value.

In order to avoid the production of identical sequences from identical startups, it is necessary to prime the random number generator. In the present program, this is accomplished by having the user type in an arbitrary four-digit number on the first use of the program each time it has been reloaded. The random number generator is then primed by being called repeatedly as many times as the number that was typed.

\section{CONCLUSIONS}

This program is currently being used in three different psychological laboratories to produce punched paper tapes to control experiments. There are probably many other laboratories where such a program would be very useful if a small computer, such as the PDP-8, were available for occasional use. The ability to produce complex paper tapes using such a program can significantly increase the capacity and versatility of existing control equipment. 1

\section{REFERENCES}

BRADY, $P, T$ : $A$ pseudo random number generator. Digtal Equipment Computer Users Society Program No. 5-25, 1965. Digital Equipment Computer Users Society, 146 Main Street. Maynard, Massachusetts 01754 .

GRIFFITH, G. A. A pseudo random number generator for the PDP-8 for use with FOCAL. Digital Equipment Computer Users Society Program No. FoCAL-1, 1969. Digital Equipment Computer Users Society, 146 Main Street, Maynard, Massachusetts 01754.

\section{NOTE}

1. The binary tapes required for running this program and an operating manual are available from the first author at the Department of Psyehology, Princeton University, Princeton, New Jersey 08540. 